\title{
Impact of Opioid Toxicity on Workplace Productivity
}

\section{(Review Article)}

\author{
By \\ EI Safty AMK and Fouad MM \\ Department of Occupational and Environmental Medicine, Faculty of Medicine, \\ Cairo University, Egypt
}

Corresponding author: Fouad MM : marwa.fouad@kasralainy.edu.eg

\begin{abstract}
Opioids are derived from opiates they include also synthetic and semi-synthetic drugs. Opioids have both recreational and medical uses. Opioid abuse is a worldwide problem with numerous and increasing mortality due to overdose. Opioids act on the opiate receptors as a potent mu receptor agonist resulting in a complex intracellular signals leading to dopamine release causing euphoria, and pain signal blocking. In cases of overdose, there is an excessive effect on respiratory center, resulting in respiratory depression and eventually death. Opioids can lower the perception of pain and in some cases decrease the pain stimulus. Stimulation of opiate receptors results in suppression of pain sensation. Opioids have an extremely wide diversity of durations of action, the intensity and quality of response to opioids can vary significantly between patients. Diagnosis of acute opioid toxicity is mainly clinically and naloxone is the cornerstone of therapy. Opioid addiction traditional treatment includes setting of counseling and mentorship besides drug treatment. Opioid prescription for pain management can have a major impact on future development of opioid use disorder but with careful monitoring and patient education about the potential opioid toxicity and risk of addiction to overcome addiction or overdose development. Opioid addiction has deep impact on workforce productivity in the form of increased absenteeism rate, decreased productive hours and higher rate of work place injuries that lead to economic burden. Our aim is studying the approach for preventing drug abuse and supporting drug users to get their lives back on track and boost workforce productivity.
\end{abstract}

Keywords: Opioids, Overdose, Addiction, Opioid prescription and Workplace productivity. 


\section{Introduction}

Papaver somniferum (named poppy plant) is a natural source from which opiate is extracted. Opiates have recreational and medical uses. Opioid drugs can be synthetic or semi-synthetic derived from opiates. Opioid abuse is a universe delinquent with plentiful and increasing mortality especially due to overdose (Dart et al., 2015).

\section{Epidemiology}

Mortality rates increased significantly in the United States between 2000 and 2014 due to overdose of opioids and opiates, with marked death rate increase in 2014 from opiates, particularly heroin (Ciejka et al., 2016). In the Middle East, South East and Asia opium dependence has been a common substance related disorder. Many countries abuse opium including Thailand, Bangladesh, Nepal, Sri Lanka, Turkey, Iraq, Jordan, Egypt, Bahrain, Oman and Kuwait. (WHO/ EMRO, 2004). According to the WHO (WHO, 2010), the prevalence of drug dependence in individuals between 15 and 64 years of age in Egypt was $0.8 \%$. Opioids were the substances of major problem in $44 \%$ of the substance dependence cases (tramadol tablets were the main dependence substances in $30 \%$ of the sample, heroin in $12 \%$, and nalbuphine in 2\%) (Mohamed et al., 2015). Opioid addiction has deep impact on workforce productivity in the form of reduced productive hours, work place injuries and high rate of absenteeism that all lead to economic burden (Florence et al., 2016).

\section{Mechanism of action:}

The opioid drugs are analogous to the three endogenous opioid peptides families: enkephalins, endorphins, and dynorphin. The opioid receptors (Mu, kappa and delta) are structurally similar but distinct in their locations and clinical effects. Each consists of seven transmembrane segments, with amino acid and carboxy termini. All opioid receptors are coupled to $\mathrm{G}$ proteins, but they use a diversity of signal transduction mechanisms that includes reducing the capability of adenylate cyclase to form cAMP, closing calcium channels that reduce the signal to release neurotransmitters, or opening potassium channels to hyperpolarize the cell thus modulating the neurotransmitters release (Waldhoer et al., 2004).

Opioid receptors exist throughout the central and peripheral nervous system and are linked to a variety of neurotransmitters, they are also located in gut. The analgesic effects of opioids result from inhibition of nociceptive signals at multiple points 
of its transmission from the peripheral nerve to spinal cord to brain. Effects on noradrenergic neurons in the locus ceruleus result in anxiolysis. Increased dopamine released in the mesolimbic system causes euphoria (Bonci et al., 2003).

\section{Pathophysiology}

Opioids can be administered through diverse routes as intravenous, intramuscular, or oral. Different routes of administration can provide different onsets of action. In case of abuse, sniffing is also a route of administrations beside the injection route. Complications of injections in abuse are common as cellulitis, abscess, thrombophlebitis, endocarditis, compartment syndrome, foreign body, human immunodeficiency virus and hepatitis (Matic et al., 2017).

Opioids act on the opiate receptors as a potent mu receptor agonist. This results in a complex intracellular signals leading to dopamine release causing euphoria, and pain signal blocking. In overdose, there is an excessive effect on respiratory center, resulting in respiratory depression and eventually death. Typical symptoms seen in overdose are in the form of pinpoint pupils, respiratory depression, and decreased conscious level, known as "opioid overdose triad."
Opioids may be agonists, partial agonists, or agonist-antagonists to opioid receptors. Opiates lower the perception of pain and in some cases decrease the pain stimulus. When opiate receptors are stimulated, it results in the suppression of pain sensation. However, not all opiate receptors have the same analgesic potency when stimulated. Opioids reduce pain perception by inhibition of synaptic neurotransmission and binding of opioid receptors in central and peripheral nervous systems.

The main opioid receptors mediating opioid effects are mu, kappa, and delta.

$\mathrm{Mu}$ receptors: analgesia, sedation, euphoria, gastrointestinal dysmotility, physical dependence and respiratory depression. $\mathrm{Mu}$ receptors cause a medullary diminished response to hypercarbia beside a respiratory response decrease to hypoxia which is the cause of respiratory depression.

- Kappa receptors: analgesia, dysphoria, diuresis, and miosis.

- Delta receptors: analgesia, cough suppression and inhibition of dopamine release. 
Sigma and delta receptors role has not been fully explained. However, sigma receptor stimulation can cause hallucinations, dysphoria, and psychosis, meanwhile delta receptor produces analgesia, euphoria, and seizures. Sigma receptors are no longer considered opioid because naloxone does not antagonize them.

Tolerance occurs rapidly with opioids. With overdose, patients often undergo respiratory failure. Tolerance to loss of the hypercarbic respiratory drive takes longer to develop than other euphoric effects, but opioid-tolerant patients do not develop complete tolerance to loss of hypoxic stimulus to respiration. This makes them susceptible to death in case of overdose (Ellis et al., 2019).

\section{Toxicokinetics}

Opioids have an extremely wide diversity of durations and intensities of effect. Alfentanil, for example, has a half-life of nearly 1.5 hours; while, Methadone has a half-life ranging between 8 to 60 hours. Opioid uptake and effect can also vary by route of administration, as patches of fentanyl or oral formulations of long-acting oxycodone and morphine. Diphenoxylate and loperamide, have almost no effect other than bowel motility suppression. Opioids such as methadone can significantly prolong the QT interval. Opioids can sometimes precipitate serotonin syndrome, especially when given to patients already taking a variety of psychoactive medications (antidepressant medications like SSRI). The intensity and quality of response to opioids can vary significantly between patients who can be unrelated to tolerance and it may be related to genetics (Cobaugh et al., 2014).

\section{History and physical examination in case of overdose:}

Patients with opioid overdose typically have decreased responsiveness, hypopnea, slow speech, and constricted pupils. Constricted pupils may be seen in opioid tolerating individuals during active use even without the associated sedation and decreased respiratory drive (Pergolizzi et al., 2008). Constipation is common, particularly in chronic consumers and the elderly. Decreased bowel motility can develop. Track marks can be seen in intravenous users, that are very small abrasion-type skin changes overlying veins, usually in the extremities but occasionally in the neck and other anatomic locations (Chou et al., 2017). 
Management of overdosed patients:

Diagnosis of acute opioid toxicity is mainly clinically. The patient develops hypopnea that can progress to apnea. Naloxone is the mainstay of therapy. The first-line treatment is airway control and rescue breathing. Adequate intravenous access is necessary so enough fluids and medication can be administered. An initial intravenous dose of 0.4 to $0.8 \mathrm{mg}$ of naloxone will quickly reverse neurologic and cardiorespiratory symptoms (King et al., 2011) although in some cases much higher doses are necessary, with case reports as high as $100 \mathrm{mg}$ of naloxone required for successful resuscitation (Caffrey and Lank, 2018). Bag-valve mask ventilation should be initiated immediately to restore oxygen supply to vital organs while evaluating available methods of naloxone administration. Basic and advanced Life Support principles should be followed during the resuscitation of the opioid poisoned patient. Laboratory testing can include drug screening (Blondell et al., 2013).

\section{Management of opioid addiction}

To qualify opioid use disorder, a patient must express at least some of the following features: longer consumption than expected, inability to tolerate reduced dosing, a great deal of time spent in pursuit of opioids, craving, use interferes with social obligations, use causes interpersonal problems, physically hazardous use, continued use despite pathologic consequences of use, tolerance causing a need for increased dosing for similar effects, and characteristic withdrawal state when use is reduced or stopped. Detoxification to the point of abstinence from all forms of opioids, including avoidance of medications as buprenorphine or methadone, does not seem to provide the same relapse prevention advantage (Saloner et al., 2018).

Traditional treatment of opioid addiction focuses on self-help in the setting of counseling and mentorship by addicts already successfully in recovery. The concept of harm reduction became increasingly accepted by the mainstream of addiction treatment providers, which allowed for ingested medications to be taken. Chronic treatment of addicts with methadone (full mu receptor agonist), buprenorphine (partial agonist of mu receptors and Kappa antagonist), and Naltrexone (opioid receptor antagonist) became more accepted, they can be used to stabilize opioid use 
disordered patients and make relapse and future opioid toxicity/overdose events less likely. Concurrently, depot naltrexone injection for enhancement of complete opioid avoidance became available. Every single naltrexone injection persists nearly 30 days. During that time, opioids became ineffective by the effect of the naltrexone on the target receptors (Sehgal et al., 2012). Disadvantages of naltrexone include difficulties in controlling acute pain in case of trauma and other acute medical issues. Depot naltrexone injection is contraindicated in the setting of chronic pain. Oral naltrexone is taken daily but is ineffective if the patient controls their dosing schedule. Observed controlled oral naltrexone intake may have promise regarding disulfiram in alcoholism management (Sporer, 1999).

\section{Differential diagnosis}

If the classic triad of opioid toxicity is present in a comatose patient and if after naloxone reversal of comma appears, opioid toxicity assumption can be justified. However, undifferentiated comatose patients are seen often in the emergency setting. Traditionally, "coma cocktail" of 4 medications (dextrose/ thiamine/naloxone/flumazenil) can be used in emergency care (Wanger et al., 1998). It is often best to consider a very small test dose of naloxone such as $0.4 \mathrm{mg}$ intravenous in the adult patient, if that medication is to be given diagnostically, and to keep the differential broad regarding potential etiologies of unresponsiveness until the history and physical examination constrict that differential.

\section{Complications of opioids}

Opioid overdose can lead to severe permanent disability or death by hypoxic organ injury. Chronic use of opioids can result in tolerance and risk for opioid use disorder. Illicit use can result in a diversity of complications, largely related to injection, including endocarditis with its further consequences. Body packers delivering potent opioids for the illicit market can have packets undergo enteral rupture, resulting in sudden, often catastrophic, loss of respiratory drive and death (Metfessel et al., 2019).

\section{Opioid prescription}

It has become increasingly clear that outpatient opioid prescription duration can have a major impact on future 
development of opioid use disorder, especially if opioid consumption continues longer than 5 days past the date of injury, with increasing risk as subsequent days pass and opioid consumption for the pain related to the injury has not yet terminated. Some studies suggest that NSAIDs work as well for acute pain as opioids, but others have suggested there may be confounding by genetic opioid response heterogeneity (Melanson et al., 2010).

In acute setting, receiving opioids for a very short duration is not likely to induce a substance use disorder. Once the opioid wears off, patients are predicted to regain normal physiology. Although there are claims that single dose of opioids created a cascade of use disorder, but it is quite rare. The more opioid doses patients receive the more chance they have for opioid use disorder. Consultation with a chronic pain management doctor should be counseled to optimize care. Gradually decreasing dosing is better than abrupt cessation of opioids in opioid prescription settings.

If there is a significant risk of misuse or overdose in the setting of chronic severe pain, prescriptions with very small numbers of doses is recommended, in some cases utilizing daily dosing of potent opioids as in patients with end stage cancer actively using heroin. Many pain management doctors feel that an opioid overdose is not itself necessarily a mandate for ceasing all outpatient opioids, but should be discussed with the patient and family regarding the risk of future overdose. It is essential for patients consuming opioids of any kind to realize the enhanced danger opioids represent when co-ingested with substances that are GABA-ergic such as alcohol, benzodiazepines or barbiturates (Gudin et al., 2013).

\section{Toxicity and side effects management in prescription opioids}

Patients who use opioids as prescribed can be at risk of overdose especially with concurrent consumption of outpatient benzodiazepines. There is similar risk enhancement if opioids and alcohol are consumed together. There are case reports of overdoses receiving successful rescue with an injection of illicit buprenorphine/naloxone, but this has been unsuccessful with layperson injection of illicit buprenorphine alone (Sivilotti, 2016). 
Suggested strategies used by physicians for prevention of opioid prescription diversion:

It is important for a provider to carefully evaluate for chronic pain in any patient. It is advised to use these ensuing strategies to avoid any opioid prescription diversion:

1. In patients who tend to overuse medications, it is often farsighted to hand out smaller quantities of medication and more frequently seeing these patients

2. Pill counts is another strategy to ensure adherence to the prescribed drug regimen

3. Ordering random urine toxicology and having a written contract to make clear the circumstances under which opioids will be stopped.

4. Prescription monitoring programs. The physician can use this program to decrease diversion by determining when a patient is receiving a prescription from multiple providers (Pauly et al., 2018).

\section{Enhancing healthcare team outcomes}

All healthcare workers who prescribe and dispense opiates are important partners in preventing the opioid overdose and risk of addiction.
All the guidelines agree that doses of opioids greater than $90-200 \mathrm{mg}$ of morphine equivalents per days should be avoided. Further, when starting or switching fentanyl patches to oral opioids, the doses should be reduced by $25-50 \%$. The guidelines also recommend the use of opioid risk assessment tools, written agreements and urine drug testing (Yokell et al., 2012). Pharmacist is perhaps in the ideal position to fight the opioid overdose when detecting high prescription doses of opioids and speaking to the healthcare provider before dispensing the drug (Bone et al., 2018).

\section{Workplace strategy for handling} addicts and increasing productivity:

Epidemiological studies revealed that more than $70 \%$ of workers with alcohol or illicit drug use continue to maintain employment and continue to remain "functioning", however over $42 \%$ report a decrease in productivity as a result, increase workplace injuries and fatalities. Organizations can no longer ignore the realities and repercussions of alcohol and other drugs in the workplace. To address this problem, the NIOSHfunded Connecticut Department of Public Health published The Opioid Crisis and Connecticut's Workforce (CDC, 2019). 
Workplace programs are more effective when an organizational, comprehensive approach is taken. These programs can reduce the risk posed by substance use in the workplace fall that can be adapted to fit with other workplace health initiatives, including interventions aimed at mental health and tobacco use (Stockwell, et al., 2005).

It is well known that workplace preparedness for dealing with drug use disorders is costly and dangerous for organizations, as well as individuals. Increasing the awareness of identifying the signs and symptoms of drug abuse, developing an employee drug testing program and investing in employee lifesaving treatment should be adopted by workplaces (Merrick et al., 20017)

\section{Conclusion}

Opioid toxicity whether in form of overdose or addiction is vital issue that needs proper assessment and management. Opioid can be used for chronic pain but only with careful monitoring and education of the patient about the potential toxicity of opioids and the risk of addiction to avert the crises of addiction or overdose. Substance abuse impact deeply work place productivity and increasing economic burden.
Recommendations:High light the workplace problem to assess the magnitude of the problem and to develop a workplace program for addiction at work place is essential, increase workplace physician awareness for their role in preventing and detection of addicts, preventing drug abuse and approach to support survivors to be back on track and enhance productivity. The organization ultimate goal should be creating a drug-free workplace.

\section{References}

1. Blondell RD, Azadfard M and Wisniewski AM (2013): Pharmacologic therapy for acute pain. Am Fam Physician; 87(11): 766-72.

2. Bonci A, Bernardi G, Grillner P and Mercuri NB (2003): The dopamine-containing neuron: maestro or simple musician in the orchestra of addiction? Trends in Pharmacological Sciences; 24(4):172-7.

3. Bone C, Eysenbach L, Bell K and Barry DT (2018): Our Ethical Obligation to Treat Opioid Use Disorder in Prisons: A Patient and Physician>s Perspective. J Law Med Ethics; 46(2): 268-71.

4. Caffrey CR and Lank PM (2018): When good times go bad: managing 'legal high'complications in the emergency department. Open access emergency medicine: OAEM; 10: 9-23. 
5. Centers for disease control and Prevention (CDC, 2019): Opioids in the Workplace: NIOSH Extramural Research. Available at https://www.cdc.gov/niosh/topics/opioids/ extramuralresearch.html

6. Chou R, Korthuis PT, McCarty D, Coffin PO, Griffin JC, et al. (2017): Management of suspected opioid overdose with naloxone in out-of-hospital settings: a systematic review. Ann Intern Med; 167(12): 867-75.

7. Ciejka M, Nguyen K, Bluth MH and Dubey E (2016): Drug toxicities of common analgesic Medications in the emergency department. Clin Lab Med; 36(4): 761-76.

8. Cobaugh DJ, Gainor C, Gaston CL, Kwong TC, Magnani B, et al. (2014): The opioid abuse and misuse epidemic: implications for pharmacists in hospitals and health systems. Am J Health-Syst $\mathrm{Ph} ;$ 71(18):1539-54.

9. Dart RC, Surratt HL, Cicero TJ, Parrino MW, Severtson SG, et al. (2015): Trends in opioid analgesic abuse and mortality in the United States. New Eng J Med; 372(3): 241-8.

10. Ellis RJ, Wang Z, Genes $\mathrm{N}$ and Ma'ayan A (2019): Predicting opioid dependence from electronic health records with machine learning. Bio Data mining; 12(1): 3.

11. Florence C, Luo F, Xu L and Zhou C (2016): The economic burden of prescription opioid overdose, abuse and dependence in the United States. Med Care; 54(10): 9016.
12. Gudin JA, Mogali S, Jones JD and Comer SD (2013): Risks, management, and monitoring of combination opioid, benzodiazepines, and/or alcohol use. Postgraduate Medicine; 125(4): 11530 .

13. King S, Forbes K, Hanks GW, Ferro CJ and Chambers EJ (2011): A systematic review of the use of opioid medication for those with moderate to severe cancer pain and renal impairment: a European Palliative Care Research Collaborative opioid guidelines project. Palliative Med; 25(5): 525-52.

14. Matic M, De Wildt SN, Tibboel D and Van Schaik RH (2017): Analgesia and opioids: a pharmacogenetics shortlist for implementation in clinical practice. Clin Chem; 63(7):1204-13.

15. Melanson SE, Baskin L, Magnani B, Kwong TC, Dizon A et al. (2010): Interpretation and utility of drug of abuse immunoassays: lessons from laboratory drug testing surveys. Arch Pathol Lab Med; 134(5): 735-9.

16. Merrick ESL, Volpe-Vartanian J, Horgan CM and McCann B (2007): Revisiting employee assistance programs and substance use problems in the workplace: key issues and a research agenda. Psychiatric services (Washington, DC); 58(10):1262. 
17. Metfessel BA, Mentel MD, Phanel A, Dimartino MA, Allen M et al. (2019): Opioid Use is Associated with Higher Severity-Adjusted Episode Costs in Patients with Conservatively Managed Degenerative Joint Disease of the Back and Neck. Pharmaco Economics; 37(3): 419-33.

18. Mohamed NR, El Hamrawy LG, Shalaby AS, El Bahy MS and Allah MMA (2015): An epidemiological study of tramadol $\mathrm{HCl}$ dependence in an outpatient addiction clinic at Heliopolis Psychiatric Hospital. Menoufia Medical Journal; 28(2): 591-6.

19. Pauly JB, Vartan CM and Brooks AT (2018): Implementation and evaluation of an opioid overdose education and naloxone distribution (OEND) program at a Veterans Affairs Medical Center. Subst Abus; 39(2): 206-10.

20. Pergolizzi J, Böger RH, Budd K, Dahan A, Erdine S, et al. (2008): Opioids and the management of chronic severe pain in the elderly: consensus statement of an International Expert Panel with focus on the six clinically most often used World Health Organization Step III opioids (buprenorphine, fentanyl, hydromorphone, methadone, morphine, oxycodone). Pain Pract; 8(4): 287-313.
21. Saloner B, McGinty EE, Beletsky L, Bluthenthal R, Beyrer C, et al. (2018): A public health strategy for the opioid crisis. Public Health Reports; 133(1_ suppl): 24S-34S.

22. Sehgal N, Manchikanti L and Smith HS (2012): Prescription opioid abuse in chronic pain: a review of opioid abuse predictors and strategies to curb opioid abuse. Pain Physician; 15(3 Suppl): ES67-ES92.

23. Sivilotti ML (2016): Flumazenil, naloxone and the 'coma cocktail'. Brit J Clin pharmaco; 81(3): 428-36.

24. Sporer KA (1999): Acute heroin overdose. Ann Intern Med; 130(7): 584-90.

25. Stockwell T, Gruenewald P, Toumbourou J, and Loxley W (Eds.) (2005): Preventing harmful substance use: The evidence base for policy and practice. Chichester, England: Wiley.

26. Waldhoer M, Bartlett SE and Whistler JL (2004): Opioid receptors. Annu Rev of Biochem; 73(1): 953-90.

27. Wanger K, Brough L, Macmillan I, Goulding J, MacPhail I and Christenson JM (1998): Intravenous vs subcutaneous naloxone for out-of-hospital management of presumed opioid overdose. Acad Emerg Med; 5(4): 293-9. 
28. WHO (2010): ATLAS of substance use disorders resources for the prevention and treatment of substance use disorders (SUD) country profile. Egypt: WHO. Available at: http://www.who.int/substance_abuse/ publications/atlas_report/profiles/egypt. pdf. [Accessed 15 April 2014]

29. WHO/EMRO (2004): WHO/EMRO. A Draft of the 5-Year Regional Strategic Planning on Substance Abuse 2005-2009. World Health Organization Department of Mental Health and Substance Abuse Management of Substance Abuse; National Drug Dependence Treatment Centre, All India. Institute of Medical Sciences New Delhi, India.

30. Yokell MA, Zaller ND, Green TC, McKenzie M and Rich JD (2012): Intravenous use of illicit buprenorphine/ naloxone to reverse an acute heroin overdose. J Opioid Manag; 8(1): 63-6. 